\title{
Stability Analysis of Pose-Based Visual Servoing Control of Cable-Driven Parallel Robots
}

\author{
Zane Zake ${ }^{12}$, Stéphane Caro ${ }^{13}$, Adolfo Suarez Roos ${ }^{2}$, François Chaumette ${ }^{4}$, \\ and Nicolò Pedemonte ${ }^{2}$ \\ 1 Laboratoire des Sciences du Numérique de Nantes, UMR CNRS 6004, \\ 1, rue de la Noë, 44321 Nantes, France, \\ Zane.Zake@ls2n.fr, \\ 2 IRT Jules Verne, Chemin du Chaffault, 44340, Bouguenais, France \\ nicolo.pedemonte@irt-jules-verne.fr, \\ 3 Centre National de la Recherche Scientifique (CNRS), \\ 1, rue de la Noë, 44321 Nantes, France, \\ stephane.caro@ls2n.fr \\ 4 Inria, Univ Rennes, CNRS, IRISA, Rennes, France, \\ Francois.Chaumette@inria.fr,
}

\begin{abstract}
Cable-driven parallel robots are robots with cables instead of rigid links. The use of cables introduces advantages such as high payload to weight ratio, large workspaces, high velocity capacity. Cables also bring drawbacks such as bad accuracy when the robot model is not accurate. In this paper, a visual servoing control is proposed in order to achieve high accuracy no matter the robot model precision. The stability of the solution is analyzed to determine the tolerable perturbation limits. Experimental validation is performed both in simulation and on a real robot to highlight the differences.
\end{abstract}

Keywords: cable-driven parallel robots, visual servoing, stability

\section{Introduction}

In cable-driven parallel robots (CDPRs) rigid links are substituted by flexible cables. This substitution leads to advantages such as: $(i)$ large workspace (WS), (ii) reconfigurability [1, and (iii) high payload to weight ratio. However, the accuracy of CDPRs is to be improved. Previously, the following methods to improve accuracy have been studied: $(i)$ increasing the complexity of the CDPR model in model-based control 2]; using proprioceptive sensors, such as (ii) force sensors to measure cable tensions $[3$ or (iii) angular position sensors to measure cable angles [4]; using exteroceptive sensors, namely vision sensors [5] [6] [7].

The increasing popularity of vision-based control is due to its high robustness to unexpected change in the environment. The two main approaches of such control are: image-based visual servoing (IBVS) and pose-based visual servoing (PBVS) [8]. The latter is used when information from the image and some additional knowledge about the object (usually its model) allows us to estimate 
the 3D pose of the object in the camera frame $\mathcal{F}_{c}$. The control consists of minimizing the difference between the acquired object pose and the desired one at each iteration. IBVS is used when estimation of the 3D pose is not possible. Here, visual features such as 2D image coordinates or image moments are used instead. The control then consists of minimizing the error in the image space by comparing the desired and the current visual features.

Vision-based control on CDPRs is not yet well researched. It is surprising given the challenge to achieve accurate model-based control, since it requires to predict complex aspects like cable elongation and sagging, and pulley effect. Furthermore, to find the solutions to the Forward Kinematic problem for CDPRs is a tedious task that requires a good knowledge of actual cable lengths and tensions, acquired by proprioceptive sensors. Although the addition of proprioceptive sensors can increase the robot accuracy, vision-based control is inherently robust to modeling errors and uncertainties, and avoids the need of computing the Forward Kinematic problem. This is attained by actively perceiving either the MP with a static camera in the so-called eye-to-hand configuration [5] [6], or the object of interest with a moving camera mounted on MP in the eye-in-hand configuration [7. The robot is then actuated according to what is perceived. Dallej et al. used multiple static cameras facing the MP as well as observing cable exit points to determine their sag [5. Multiple control schemes were proposed. Similarly, Chellal et al. used 6 infra-red sensors to determine the MP pose with high accuracy [6. Remy et al. used the eye-in-hand configuration with a single camera [7. Their task was to control a spatial CDPR with three translational degrees of freedom. Finally, a control scheme was proposed in 9 to control all six degrees of freedom while still using only one camera mounted on the MP.

One of the main characteristics of any control scheme is its stability. It was found in [9] that the PBVS control of a CDPR is highly robust to modeling errors. However due to the wide range of such errors and their combined effect on the system it was not possible to determine their maximum values. In this paper, possible sources of errors are studied separately to find their maximum values for different MP working range. Such a separated analysis does not allow us to evaluate the interaction between different errors. For this reason, a second evaluation is done, assuming a constant non-negligible perturbation. These values are experimentally validated both in simulation and on a CDPR prototype.

This paper is organized as follows. Section 2 presents the control scheme. Stability condition is established in Section 3. A case study is shown in Section 4 . Finally, the conclusions are drawn in Section 5.

\section{Vision-Based Control of a CDPR}

\subsection{CDPR Kinematics}

The schematic of a spatial CDPR in a suspended configuration is shown in Fig. 1.

Given that the camera is mounted on the MP, the transformation matrix

${ }^{p} \boldsymbol{T}_{c}$ between the camera frame $\mathcal{F}_{c}$ and the MP frame $\mathcal{F}_{p}$ is constant. On the contrary, the transformation matrices ${ }^{b} \boldsymbol{T}_{p}$ and ${ }^{c} \boldsymbol{T}_{o}$ change with time. 


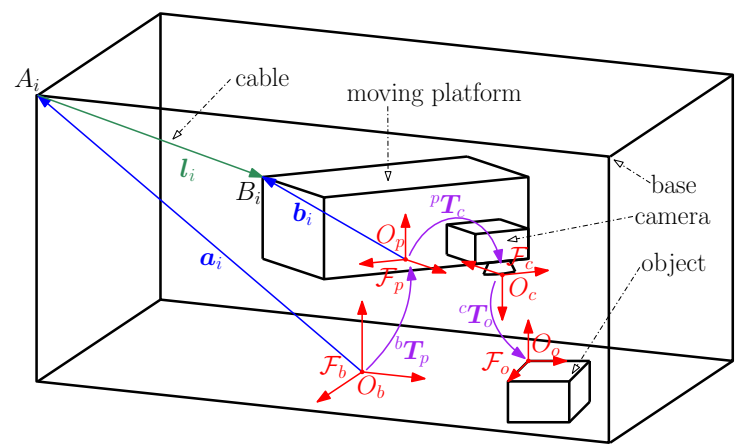

Fig. 1. Schematic of a spatial CDPR with eight cables, a camera mounted on its MP and an object in the WS.

The length $l_{i}$ of the $i$ th cable is the 2-norm of the vector $\overrightarrow{A_{i} B_{i}}$ pointing from cable exit point $A_{i}$ to cable anchor point $B_{i}$, namely,

$$
l_{i}=\left\|\overrightarrow{A_{i} B_{i}}\right\|_{2}
$$

with

$$
l_{i}{ }^{b} \mathbf{u}_{i}={ }^{b} \overrightarrow{A_{i} B_{i}}={ }^{b} \boldsymbol{b}_{i}-{ }^{b} \boldsymbol{a}_{i}={ }^{b} \boldsymbol{R}_{p}{ }^{p} \boldsymbol{b}_{i}+{ }^{b} \boldsymbol{t}_{p}-{ }^{b} \boldsymbol{a}_{i}
$$

where ${ }^{b} \mathbf{u}_{i}$ is the unit vector of ${ }^{b} \overrightarrow{A_{i} B_{i}}$ that is expressed as:

$$
{ }^{b} \mathbf{u}_{i}=\frac{{ }^{b} \overrightarrow{A_{i} B_{i}}}{\left\|{ }^{b} \overrightarrow{A_{i} B_{i}}\right\|_{2}}=\frac{{ }^{b} \boldsymbol{b}_{i}-{ }^{b} \boldsymbol{a}_{i}}{\left\|{ }^{b} \overrightarrow{A_{i} B_{i}}\right\|_{2}}=\frac{{ }^{b} \boldsymbol{R}_{p}{ }^{p} \boldsymbol{b}_{i}-{ }^{b} \boldsymbol{a}_{i}+{ }^{b} \boldsymbol{t}_{p}}{\left\|b \overrightarrow{A_{i} B_{i}}\right\|_{2}}
$$

The cable velocities $i_{i}$ are obtained upon differentiation of Eq. (2) with respect to (w.r.t.) time:

$$
i=\boldsymbol{A}^{b} \mathbf{v}_{p}
$$

where ${ }^{b} \mathbf{v}_{p}$ is the Cartesian velocity of the MP expressed in $\mathcal{F}_{b}, \boldsymbol{i}$ is the cable velocity vector, and $\boldsymbol{A}$ is the Forward Jacobian matrix of the CDPR, defined as [10]:

$$
\boldsymbol{A}=\left[\begin{array}{cc}
{ }^{b} \mathbf{u}_{1}^{T} & \left({ }^{b} \boldsymbol{R}_{p}{ }^{p} \boldsymbol{b}_{1} \times{ }^{b} \mathbf{u}_{1}\right)^{T} \\
\vdots & \vdots \\
{ }^{b} \mathbf{u}_{m}^{T} & \left({ }^{b} \boldsymbol{R}_{p}{ }^{p} \boldsymbol{b}_{m} \times{ }^{b} \mathbf{u}_{m}\right)^{T}
\end{array}\right]
$$

\section{$2.2 \quad$ Pose-Based Visual Servoing}

The control scheme proposed in this paper is shown in Fig. 2. An image is retrieved from the camera and processed with a computer vision algorithm, that returns the current pose of the object $s$. It is compared to a previously 
known desired pose $\boldsymbol{s}^{*}$ 场 and an error vector $\boldsymbol{e}$ is defined as $\boldsymbol{e}=\left[\boldsymbol{e}_{t}^{T} \boldsymbol{e}_{\omega}^{T}\right]^{T}$, where $\boldsymbol{e}_{t}={ }^{c} \boldsymbol{t}_{o}-{ }^{c^{*}} \boldsymbol{t}_{o^{*}}=\left[\begin{array}{lll}e_{x} & e_{y} & e_{z}\end{array}\right]^{T}$ and $\boldsymbol{e}_{\omega}=\mathbf{u} \theta$, $\mathbf{u}$ being the axis and $\theta$ the angle of the rotation matrix ${ }^{c} \boldsymbol{R}_{c^{*}}$.

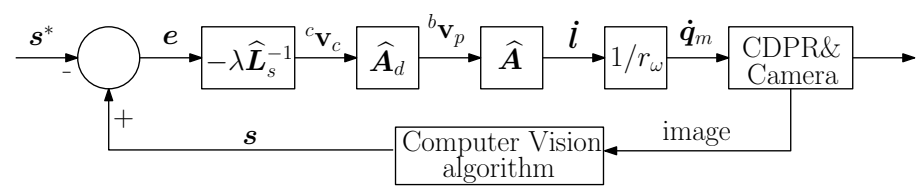

Fig. 2. Control scheme for pose-based visual servoing of a CDPR

To decrease the error $\boldsymbol{e}$, an exponential decoupled form is selected $\dot{\boldsymbol{e}}=-\lambda \boldsymbol{e}$ with a positive adaptive gain $\lambda$, that is computed at each iteration, depending on the current $\|\boldsymbol{e}\|_{2}[7$.

The relationship between $\dot{e}$ and the Cartesian velocity of the camera ${ }^{c} \mathbf{v}_{c}$, expressed in $\mathcal{F}_{c}$, is defined as:

$$
\dot{e}=L_{s}{ }^{c} \mathbf{v}_{c}
$$

where $\boldsymbol{L}_{\boldsymbol{s}}$ is the interaction matrix and it is defined in 8 :

Finally, the instantaneous velocity of the camera in its own frame is expressed as a function of the pose error as follows:

$$
{ }^{c} \mathbf{v}_{c}=-\lambda \widehat{\boldsymbol{L}}_{\boldsymbol{s}}^{-1} \boldsymbol{e}
$$

where $\widehat{\boldsymbol{L}}_{\boldsymbol{s}}^{-1}$ is the inverse of the estimation of the interaction matrix $\widehat{\boldsymbol{L}}_{\boldsymbol{s}}$. Note that the inverse is directly used, because for PBVS $\boldsymbol{L}_{\boldsymbol{s}}$ is a $(6 \times 6)$-matrix that is of full rank [8.

\subsection{Kinematics and Vision}

To combine the modeling shown in Sects. 2.1 and 2.2. the MP twist ${ }^{b} \mathbf{v}_{p}$ is expressed as a function of camera velocity ${ }^{c} \mathbf{v}_{c}$ :

$$
{ }^{b} \mathbf{v}_{p}=\boldsymbol{A}_{d}{ }^{c} \mathbf{v}_{c}
$$

where $\boldsymbol{A}_{d}$ is the adjoint matrix that takes the following form [11]:

$$
\boldsymbol{A}_{d}=\left[\begin{array}{cc}
{ }^{b} \boldsymbol{R}_{c} & \left.{ }^{b} \boldsymbol{t}_{c}\right]_{\times}{ }^{b} \boldsymbol{R}_{c} \\
\mathbf{0}_{3} & { }^{b} \boldsymbol{R}_{c}
\end{array}\right]
$$

where ${ }^{b} \boldsymbol{t}_{c}={ }^{b} \boldsymbol{R}_{p}{ }^{p} \boldsymbol{t}_{c}$ and ${ }^{p} \boldsymbol{t}_{c}$ is the vector pointing from $O_{p}$ to $O_{c}$.

\footnotetext{
${ }^{5}$ In this paper, a superscript $*$ denotes the desired value, e.g. desired object pose $\boldsymbol{s}^{*}$ and $c^{*}$ in ${ }^{c^{*}} \boldsymbol{t}_{o^{*}}$ refers to desired camera frame $\mathcal{F}_{c^{*}}$ in which the object is in the desired pose $\boldsymbol{s}^{*}$
} 


\section{Stability Condition}

One of the main characteristics of any system is its stability. It is a measure to assess the effects of estimation quality. That is, how coarse can the estimation be for the system to still converge to its goal [12. Lyapunov analysis is used to determine the stability of the closed-loop visual servoing system.

From Eqs. (4), (6) and (8) the model is the following:

$$
\dot{e}=L_{s} A_{d}^{-1} A^{\dagger} i
$$

Upon injecting (8) and (7) into (4), the output of the control scheme, that is, the cable velocity vector takes the form:

$$
i=-\lambda \widehat{A} \widehat{A}_{d} \widehat{\boldsymbol{L}}_{\boldsymbol{s}}^{-1} \boldsymbol{e}
$$

where $\widehat{\boldsymbol{A}}$ and $\widehat{\boldsymbol{A}}_{d}$ are the estimations of $\boldsymbol{A}$ and $\boldsymbol{A}_{d}$, respectively.

The following closed-loop equation is obtained from (10) and (11):

$$
\dot{\boldsymbol{e}}=-\lambda \boldsymbol{L}_{\boldsymbol{s}} \boldsymbol{A}_{d}^{-1} \boldsymbol{A}^{\dagger} \widehat{\boldsymbol{A}} \widehat{\boldsymbol{A}}_{d} \widehat{\boldsymbol{L}}_{\boldsymbol{s}}^{-1} \boldsymbol{e}
$$

From 12 , the system stability criterion is defined as:

$$
\boldsymbol{\Pi}=\boldsymbol{L}_{\boldsymbol{s}} \boldsymbol{A}_{d}^{-1} \boldsymbol{A}^{\dagger} \widehat{\boldsymbol{A}} \widehat{\boldsymbol{A}}_{d} \widehat{\boldsymbol{L}}_{\boldsymbol{s}}^{-1}>\mathbf{0}, \forall t
$$

$\boldsymbol{\Pi}>\mathbf{0}$ is a sufficient condition to obtain global asymptotic stability (GAS). It can be seen from the closed-loop equation (12) that if $\boldsymbol{\Pi}$ is positive definite, then the control scheme will ensure an exponential convergence of the error $\boldsymbol{e}$ to $\mathbf{0}$. However, if it is negative then the error $\boldsymbol{e}$ will increase and the system may diverge from the goal. Indeed, $\sqrt{13}$ is only a sufficient condition, therefore the stability of the system is uncertain once the condition is not held.

\subsection{Estimated Parameters}

Given the closed loop equation 12 , the following variables are estimated and can therefore affect the system stability:

- $\hat{\boldsymbol{s}}$ - object pose in $\mathcal{F}_{c}$ is computed from image features, so it will not be the exact pose $s$

${ }^{p} \widehat{\boldsymbol{T}}_{c}$ - the pose of the camera in the MP frame $\mathcal{F}_{p}$. We have an idealistic model, however due to manufacturing imprecisions, the actual camera pose will be a little bit different from the modeled pose.

${ }^{p} \widehat{B}_{i}$ - the Cartesian coordinates of cable anchor points to the MP, expressed in $\mathcal{F}_{p}$. Due to mechanical solution of the anchor points, the actual point is a point located on a sphere around the nominal point.

$-{ }^{b} \widehat{A}_{i}$ - the Cartesian coordinates of cable exit points, expressed in the base frame $\mathcal{F}_{b}$. We are using the simplified CDPR model, that does not include pulleys actually located at the exit points. 


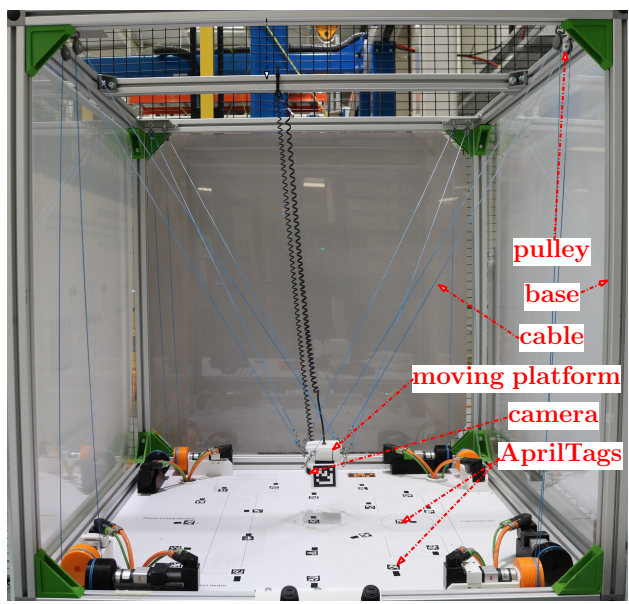

(a)

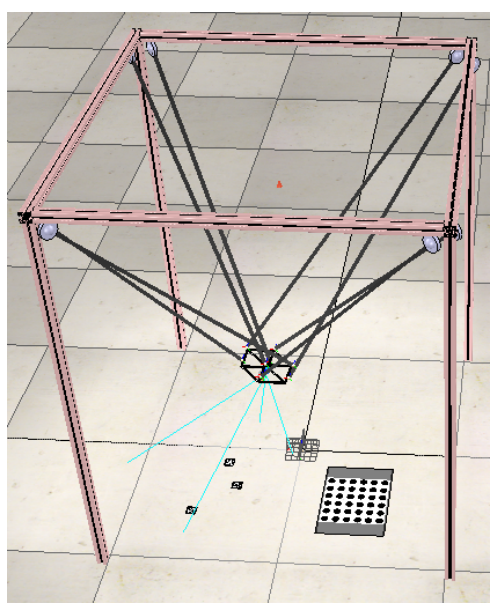

$(b)$

Fig. 3. CDPR prototype: (a) ACROBOT; (b) V-REP model of ACROBOT

$-{ }^{b} \widehat{\boldsymbol{T}}_{p},{ }^{b} \boldsymbol{T}_{p}$ is estimated by exponential mapping:

$$
\left({ }^{b} \boldsymbol{T}_{p}\right)_{t+\Delta t}=\left({ }^{b} \boldsymbol{T}_{c}\right)_{t+\Delta t}{ }^{c} \boldsymbol{T}_{p}=\left({ }^{b} \boldsymbol{T}_{c}\right)_{t} \exp \left({ }^{c} \mathbf{v}_{c}, \Delta t\right){ }^{c} \boldsymbol{T}_{p}
$$

Since velocity ${ }^{c} \mathbf{v}_{c}$, necessary for exponential mapping, is computed from the object pose measurement $\hat{\boldsymbol{s}}$, which we admit to be different from $\boldsymbol{s}$, then ${ }^{b} \widehat{\boldsymbol{T}}_{p} \neq{ }^{b} \boldsymbol{T}_{p}$. Furthermore, initial ${ }^{b} \boldsymbol{T}_{p}$ is only coarsely known 6

\section{Case Study}

The stability condition (13) is applicable to any CDPR with a PBVS control. However, this analysis includes model-related parameters, thus it should be done for each CPDR separately. Hence, in the following sections the chosen CDPR is presented and results of stability analysis are shown.

\subsection{ACROBOT and Simulation in V-REP}

ACROBOT For this paper, a CDPR prototype, named ACROBOT and shown in Fig. 3(a), is used. Its WS is a $1 \mathrm{~m}^{3}$ cube. The robot is assembled in a suspended configuration. A simple webcam AUTOPIX MT4018 is mounted on the MP in the eye-in-hand configuration facing the ground.

To simplify the computer vision part, AprilTags [14] are used instead of real objects. They are especially convenient to use in combination with ViSP library [15, because the latter contains functions that allow to recognize the tags and retrieve their $3 \mathrm{D}$ pose.

\footnotetext{
${ }^{6}$ For this CDPR, the initial pose was defined at the center of the WS, which itself was
} measured by hand with a measurement error of $\pm 2 \mathrm{~cm}$ along $\mathrm{X}$ and $\mathrm{Y}$ axes, resp. 


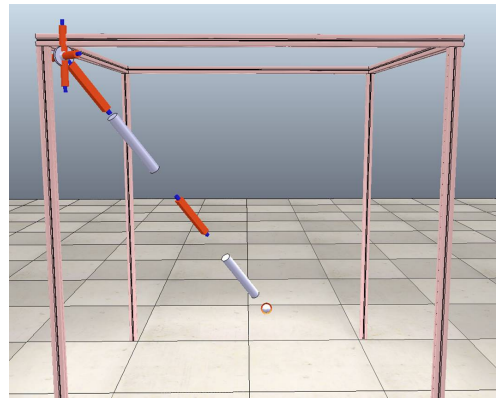

Fig. 4. The representation of a cable and its pulley in V-REP

V-REP Model The V-REP simulation environment [16 was used in order to create a dynamic model of ACROBOT including the vision sensor. This gives us the capacity to use the same software to control the real and the virtual hardware. As a result we can speed up the development time, test and debug our algorithms in simulation (with a perfectly known ground truth), then use the real robot for final verifications.

To create a dynamical simulation, the pulleys and cables are modeled as a sequence of joints and mass objects, as shown in Fig 4 . The current model does not take into account the pulley diameter and the cable sag. The pulleys are represented as a vertical revolute passive joint followed by a small spherical mass and a horizontal revolute passive joint. The cables are modeled as a sequence of prismatic joint, cylindrical mass, prismatic joint, cylindrical mass and a final spherical joint attaches the cable to the MP. The first prismatic joint is used to change the cable length. The second prismatic joint is responsible for the cable behavior through a specific joint control callback script, which models the cable forces as either an elastic spring, when in tension, or an element transmitting zero force, when in compression.

To have a stable simulation some model design rules need to be considered [17. In our tests, the Vortex physical engine was used.

\subsection{Numerical Analysis}

It is not possible to express analytically the pseudo-inverse of the Jacobian $\boldsymbol{A}^{\dagger}$, thus stability analysis is only possible in numerical form. In this paper, we will only study two parameters, namely ${ }^{p} \boldsymbol{T}_{c}$ and ${ }^{b} A_{i}$.

The Cartesian coordinates of ${ }^{b} A_{i}$ are known, we need to find out the range of perturbation that does not destabilize the system. In Fig. $5(a)$ the perturbation range is defined by the radius $r_{A i}$ of the sphere centered at point $A_{i}$. We want to find the maximum value $r_{A i, \max }$ so that for any point within a sphere of radius $r_{A i} \leq r_{A i, \max }$ the system is stable.

Unlike cable exit points, camera pose in $\mathcal{F}_{p}$ could in fact be changed, if necessary. Therefore, here we first define the range, where the camera could 


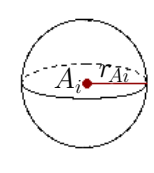

(a)

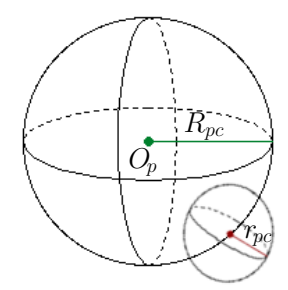

(b)

Fig. 5. Perturbation ranges for: $(a)$ cable exit points $A_{i} ;(b)$ camera pose in $\mathcal{F}_{p}$

be positioned, which is defined in Fig. $5(b)$ by radius $R_{p c}$ of the largest sphere around origin $O_{p}$ of $\mathcal{F}_{p}$. Then for any camera position, the perturbation range is defined by the small sphere radius $r_{p c}$. Our goal is to find $r_{p c, \max }$ so that if $r_{p c} \leq r_{p c, \max }$ and camera position is within the sphere defined by radius $R_{p c}$, then the system will be stable.

Finally, we transform ${ }^{p} \boldsymbol{R}_{c}$ into axis-angle representation $\theta_{p c} \mathbf{u}$. We define the perturbation as $\delta \theta_{p c}$, that can be made about any unit vector $\mathbf{u}_{\delta}$. Therefore, our goal is to find $\delta \theta_{p c, \max }$ so that $\left|\delta \theta_{p c}\right| \leq \delta \theta_{p c, \max }$ for any $\mathbf{u}_{\delta}$, $\mathbf{u}$ and $\theta_{p c}$.

To find the range of perturbation, we define the system as stated in Table 1 . Then we portray two distinct cases: (i) The system is assumed to be ideal, no perturbations other than the one we are studying; $(i i)$ the system has small perturbations in all parameters. The perturbation values are chosen either based on the mechanical errors ( $\operatorname{such}$ as ${ }^{p} B_{i}$ and ${ }^{b} A_{i}$ ) or as 5 to 10 percent of the actual parameter value. For the latter, we also distinguish the results depending on the desired range of motion of the MP in $\mathcal{F}_{b}$ expressed as $R_{b p}$.

The perturbation limits that we have established based on condition (13) are shown in Table 2 . Here are the observations:

- None of the parameters are affected by the actual value of ${ }^{p} \boldsymbol{t}_{c}$ or ${ }^{p} \boldsymbol{R}_{c}$. This is especially surprising for the perturbations on these parameters defined by $r_{p c}$ and $\delta \theta_{p c}$.

- To keep the full motion range $R_{b p}$ and the full rotational range ${ }^{b} \boldsymbol{R}_{p}$, perturbations on ${ }^{b} A_{i}$ must not be larger than $0.01 \mathrm{~m}$.

- In the ideal case, the perturbation values for camera pose in $\mathcal{F}_{p}$ are large. In fact these perturbations do not appear separately. Indeed, all the considered perturbations are affecting the system at the same time, not independently. For this reason it was important to do the second part of this study while considering perturbations in all parameters.

- When the perturbations, defined in Table 1 are added to the system, the latter remains stable for all tested motion range of MP.

- However, once the MP motion range is reduced, it is possible to increase the perturbation levels without making the system unstable.

- As soon as $R_{b p}$ is reduced, $r_{p c}$ increases significantly, as well as $\delta \theta_{p c}$. This allows to conclude that perturbations in the respective variables have little effect on the stability of the system. 
- There is a considerable increase in $r_{A i}$ as well. This means that the modeling accuracy of exit points is not important. Even at WS borders the tolerated perturbation easily covers the corresponding model inaccuracies. That is, even though pulleys that are present on the cable exit points are not modeled, the small changes in the actual exit point location on the pulley are covered by the perturbation $r_{A i}=0.01 \mathrm{~m}$ and do not affect the system stability.

Table 1. Variable and perturbation ranges

\begin{tabular}{c|c|c}
\hline variable family & variable value & perturbation value \\
\hline \multirow{2}{*}{ object pose in $\mathcal{F}_{c}$} & $R_{\boldsymbol{s}_{t}}=0.5 \mathrm{~m}$ & $r_{\boldsymbol{s}_{t}}=0.05 \mathrm{~m}$ \\
\cline { 2 - 3 } & $\left|\theta_{\omega}\right| \leq 50^{\circ}$ & $\left|\delta \theta_{\omega}\right| \leq 2.5^{\circ}$ \\
\hline \multirow{2}{*}{$\begin{array}{c}\text { camera pose in } \mathcal{F}_{p} \\
\text { cable anchor } \\
\text { points }{ }^{p} B_{i}\end{array}$} & $\begin{array}{c}\text { In eight corners of MP of } \\
\text { size } 0.1 \times 0.1 \times 0.05 \mathrm{~m}\end{array}$ & $r_{p c}=0.01 \mathrm{~m}$ \\
\hline $\begin{array}{c}\text { cable exit } \\
\text { points }{ }^{b} A_{i}\end{array}$ & $\begin{array}{c}\text { Two exit points at each top corner } \\
\text { of } \mathrm{ACROBOT}\end{array}$ & $\theta_{p c}=3^{\circ}$ \\
\hline \multirow{2}{*}{\begin{tabular}{c} 
MP pose in $\mathcal{F}_{b}$ \\
\cline { 2 - 3 }
\end{tabular}} & $\begin{array}{c}\text { Rotation about global axes: } 45^{\circ} \text { about } \mathrm{Z}, \\
20^{\circ} \text { about } \mathrm{Y}, 20^{\circ} \text { about X }\end{array}$ & $\begin{array}{c}5^{\circ} \text { about } \mathrm{Z} \text { axis, } 3^{\circ} \\
\text { about } \mathrm{Y} \text { and } \mathrm{X} \text { axes }\end{array}$ \\
\hline
\end{tabular}

Table 2. Perturbation change depending on MP motion range

\begin{tabular}{c|c|c|c}
\hline Condition & \multicolumn{2}{|c|}{ Camera pose in $\mathcal{F}_{p}$} & Cable exit points ${ }^{b} A_{i}$ \\
\hline $\begin{array}{c}\text { Ideal robot, no other perturba- } \\
\text { tion in the system, } R_{b p}=0.5 \mathrm{~m}\end{array}$ & $r_{p c}=0.5 \mathrm{~m}$ & $\left|\delta \theta_{p c}\right| \leq 55^{\circ}$ & $r_{A i}=0.01 \mathrm{~m}$ \\
\hline $\begin{array}{c}\text { Minimal perturbations from } \\
\text { Table 1. } R_{b p}=0.5 \mathrm{~m}\end{array}$ & $r_{p c}=0.03 \mathrm{~m}$ & $\left|\delta \theta_{p c}\right| \leq 3^{\circ}$ & $r_{A i}=0.01 \mathrm{~m}$ \\
\hline $\begin{array}{c}\text { Minimal perturbations from } \\
\text { Table 1. } R_{b p}=0.3 \mathrm{~m}\end{array}$ & $r_{p c}=0.5 \mathrm{~m}$ & $\left|\delta \theta_{p c}\right| \leq 16^{\circ}$ & $r_{A i}=0.09 \mathrm{~m}$ \\
\hline $\begin{array}{c}\text { Minimal perturbations from } \\
\text { Table 1. } R_{b p}=0.1 \mathrm{~m}\end{array}$ & $r_{p c}=0.8 \mathrm{~m}$ & $\left|\delta \theta_{p c}\right| \leq 24^{\circ}$ & $r_{A i}=0.18 \mathrm{~m}$ \\
\hline
\end{tabular}

\subsection{Experimental Validation}

Some experiments have been conducted to validate the theoretical results: First, we assume that the rotational error of the camera in $\mathcal{F}_{p}$ and about $\mathrm{Z}$ axis is out

${ }^{7}$ Please also see the accompanying video at https://youtu.be/tfiTDlp1ZIY 


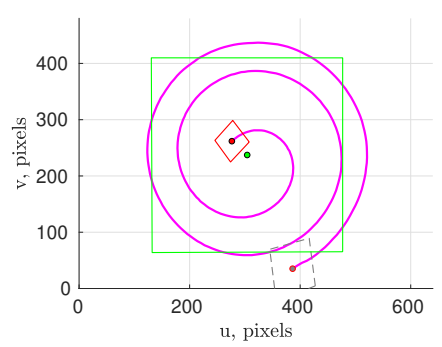

(a)

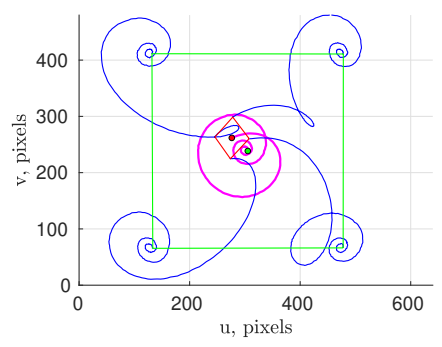

$(c)$

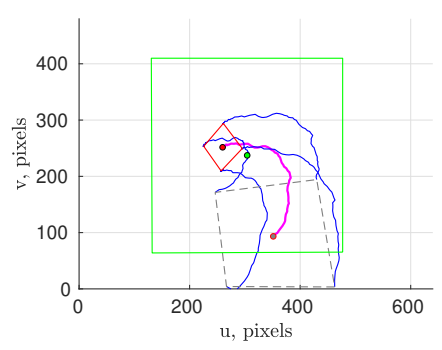

$(e)$

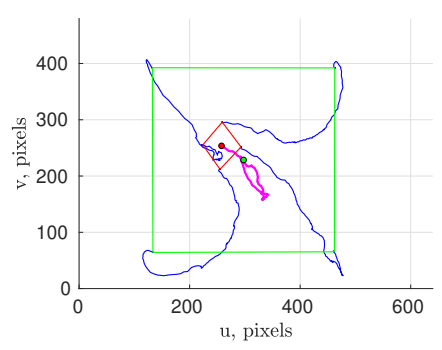

$(g)$

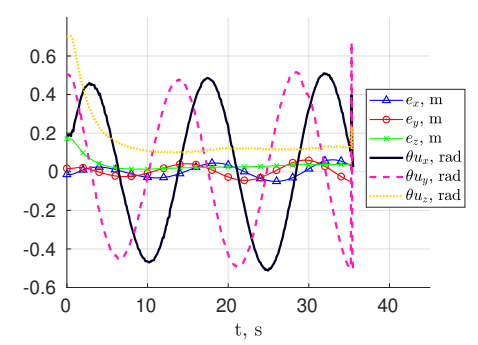

(b)

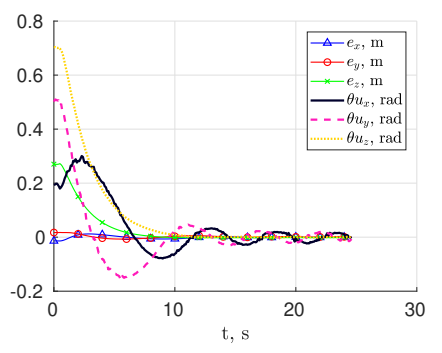

$(d)$

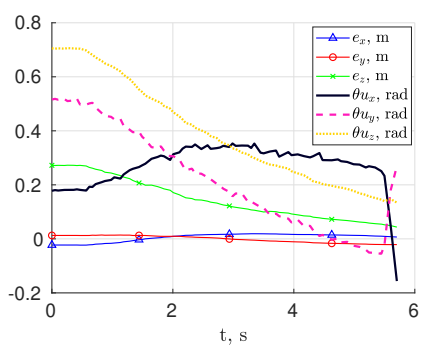

$(f)$

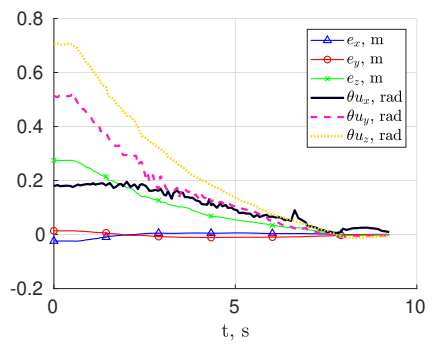

$(h)$

Fig. 6. CDPR behavior depending on added perturbations. (a) and (b): the AprilTag trajectory and error $\boldsymbol{e}$ over time in V-REP simulation with $\delta \theta=85^{\circ}$; system is not stable (only center-point trajectory shown). $(c)$ and $(d)$ : the AprilTag trajectory and error $\boldsymbol{e}$ over time in V-REP with $\delta \theta=55^{\circ}$; system is stable; $(e)$ and $(f)$ : the AprilTag trajectory and error $\boldsymbol{e}$ over time on ACROBOT with $\delta \theta=55^{\circ}$; robot does not converge because AprilTag leaves the camera field of view. $(g)$ and $(h)$ : the AprilTag trajectory and error $\boldsymbol{e}$ over time on ACROBOT with $\delta \theta=16^{\circ}$; system is stable. 
of bounds of stability, i.e. $\delta \theta_{p c}=85^{\circ}$. As shown in Fig. 6( $(a)$ the robot diverges from its goal position and error oscillates and slowly increases (Fig. 6(b)), the system is not stable.

If $\delta \theta_{p c}=55^{\circ}$, the V-REP model will successfully reach the target as shown in Fig. 6(c), though the trajectory is far from optimal (a straight line). Some error oscillations can be observed in Fig. 6(d). The same rotational error on the actual robot is shown in Fig. 6(e). The initial behavior is similar, but AprilTag leaves the image and the task is failed. This is not surprising, given the additional uncertainties of the actual robot and the lower image quality.

Finally, $\delta \theta_{p c}$ was set to its maximum for a noisy system with range of motion reduced to $R_{b p}=0.3 \mathrm{~m}$, i.e. $\delta \theta_{p c}=16^{\circ}$. Once implemented on ACROBOT, we see that though the trajectory is perturbed, the MP reaches its targeted pose (Fig. 6 $(g)$ ). Indeed, error $\boldsymbol{e}$ converges to zero without oscillations (Fig. 6( $h)$ ).

To summarize, for an ideal robot the range of perturbation on a single parameter is very large. On the contrary, as soon as we acknowledge that all the system parameters are noisy, then each individual perturbation has quite a limited range within the bounds of stability.

\section{Conclusions}

This paper proposed a method to analyze the stability of Pose-Based Visual Servoing (PBVS) control of Cable-Driven Parallel Robots (CDPRs). A general stability criterion was introduced. The stability of ACROBOT, a CDPR prototype located at IRT Jules Verne, was analyzed. Two CDPR model-related parameters were studied and their maximum perturbation range was found both for ideal robot and for a noisy one. A dynamic CDPR model in V-REP was presented.

It was found that for an ideal system any one parameter could be highly perturbed without making the system unstable. This result was successfully validated in simulation. When adding a large perturbation (validated in simulation) to ACROBOT, the system does not converge. However, if the perturbation is kept within the corresponding (noisy system) range, the robot will be able to complete its task.

For ACROBOT, the tolerated perturbation on cable exit points is rather large. This is beneficial, because it allows to avoid adding pulley kinematics to the model. Indeed, cable exit point variations on the pulley are smaller than the tolerated perturbation, which does not affect the stability of the system.

The added perturbations affect the trajectory to the goal and the system's ability to actually reach it. However, perturbations in the robot model do not affect the final accuracy. This is because the final pose is considered to be reached only when the error between the current and the desired object pose becomes smaller than the threshold defined in the visual servoing loop.

Future work will deal with the implementation of PBVS control on a large semi-industrial CDPR prototype as well as its stability analysis. It is also of interest to consider real objects instead of AprilTags to validate this approach for more realistic use cases. 
Acknowledgment This work is supported by IRT Jules Verne (French Institute in Research and Technology in Advanced Manufacturing) in the framework of the PERFORM project.

\section{References}

1. L. Gagliardini, S. Caro, M. Gouttefarde, and A. Girin, "Discrete Reconfiguration Planning for Cable-Driven Parallel Robots", in Mechanism and Machine Theory, vol. 100, pp. 313-337, 2016.

2. V. L. Schmidt, "Modeling Techniques and Reliable Real-Time Implementation of Kinematics for Cable-Driven Parallel Robots using Polymer Fiber Cables", Ph.D. dissertation, Fraunhofer Verlag, Stuttgart, Germany, 2017.

3. E. Picard, S. Caro, F. Claveau, and F. Plestan, "Pulleys and Force Sensors Influence on Payload Estimation of Cable-Driven Parallel Robots", in IROS, Madrid, Spain, October, 1-5 2018.

4. A. Fortin-Côté, P. Cardou, A. Campeau-Lecours, "Improving Cable-Driven Parallel Robot Accuracy Through Angular Position Sensors", in IROS, pp. 4350-4355, 2016.

5. T. Dallej, M. Gouttefarde, N. Andreff, R.Dahmouche, and P. Martinet, "VisionBased Modeling and Control of Large-Dimension Cable-Driven Parallel Robots", in IROS, pp. 1581-1586, 2012.

6. R. Chellal, L. Cuvillon, and E. Laroche, "A Kinematic Vision-Based Position Control of a 6-DoF Cable-Driven Parallel Robot, in Cable-Driven Parallel Robots, pp. 213-225, Springer, Cham, 2015.

7. R. Ramadour, F. Chaumette, and J.-P. Merlet, "Grasping Objects With a CableDriven Parallel Robot Designed for Transfer Operation by Visual Servoing", in ICRA, pp. 4463-4468, IEEE, 2014.

8. F. Chaumette, S. Hutchinson, "Visual servo Control I. Basic Approaches", in IEEE Robotcs \& Automation Magazine, vol. 13, no. 4, pp. 82-90, 2006.

9. Z. Zake, F. Chaumette, N. Pedemonte, S. Caro, "Vision-based Control and Stability analysis of a Cable-Driven Parallel Robot", submitted to ICRA 2019.

10. A. Pott, "Cable-Driven Parallel Robots: Theory and Application", vol. 120., Springer, 2018, pp. 52-56.

11. W. Khalil, E. Dombre, "Modeling, Identification and Control of Robots", Butterworth-Heinemann, 2004, pp. 13-29.

12. H. K. Khalil, Nonlinear systems 2nd ed., Macmillan publishing Co., New York 1996.

13. C.R. Johnson, "Positive definite matrices", in The American Mathematical Monthly, vol. 77, no. 3, pp. 259-264, 1970.

14. E. Olson, "AprilTag: A robust and flexible visual fiducial system", in ICRA, pp. 3400-3407, IEEE, 2011.

15. É. Marchand, F. Spindler, F. Chaumette, "ViSP for visual servoing: a generic software platform with a wide class of robot control skills", in IEEE Robotics 86 Automation Magazine, vol. 12, no. 4, pp. 40-52.

16. E. Rohmer, S. P. Singh, M. Freese, "V-REP: A versatile and scalable robot simulation framework", in IROS, pp. 1321-1326, 2013.

17. V-REP, Designing Dynamical Simulations. http://www.coppeliarobotics.com/ helpFiles/en/designingDynamicSimulations.htm 